\title{
DEVELOPMENT OF ANATOMICALLY CORRECT MOCK-UPS OF THE AORTA FOR PIV INVESTIGATIONS
}

\author{
Ronnie Yip ${ }^{(1)}$, Rosaire Mongrain ${ }^{(1,2)}$, Adrian Ranga ${ }^{(1)}$, Jean Brunette ${ }^{(2)}$, Raymond Cartier ${ }^{(2)}$ \\ (1) Department of Mechanical Engineering, McGill University \\ (2) Montreal Heart Institute
}

\begin{abstract}
Cardiovascular diseases are the leading cause of morbidity and mortality in Canada and other western countries. In that context, understanding the mechanics involved in the aorta can lead to better comprehension of the interaction of blood flow and wall mechanics in normal and pathologic conditions.
\end{abstract}

In this work, we present the methodologies to create physical models of the aorta for flow visualization purposes in order to assess the effects of compliance.

\section{Introduction}

The leading cause of morbidity and mortality in Canada and other Western countries is cardiovascular diseases [22]. In that context, understanding the mechanics involved in the aorta can lead to better comprehension of the interaction of blood flow and wall mechanics in normal and pathologic conditions. Indeed, by studying the fluid structure inside the compliant model of the aorta, the hemodynamics and its changes attributed to aortic diseases such as aneurysms, aortic root dilation and aortic dissections can potentially be realized. This knowledge could also be transposed to improve the designs of vascular prostheses (grafts, stents).

Few compliant models incorporating the sinus morphology, ascending aorta and aortic arch have been investigated. Development of an anatomically faithful model allows for the imaging of the fluid structure inside its complex geometries. Hemodynamic forces such as wall shear stress can be estimated based on the imaged results. Such a model can also be used to validate computational solutions of the solid and fluid mechanics involved.

Compliance in the aorta is thought to have a strong role in the progression of cardiovascular diseases. It is for this reason that the development of a rigid model is investigated in addition to its compliant counterpart.

There are several investigations that are related to the blood flow mechanics of the aorta and the $3 \mathrm{D}$ modelling of anatomical structures. They may be of either computational or experimental in nature. Three dimensional modelling of anatomical structures is often based on MRI data. For this study, the aorta model is reconstructed from MRI through image segmentations. Moore et al. [20] used a similar technique to reconstruct a carotid artery bifurcation volume mesh to perform computational steady and unsteady blood flow simulations. Moore et al. [19] also examined the errors associated with reconstructing finite element models from such data. Leuprecht et al. [17] performed physiologically correct modelling of blood flow through the human ascending aorta by combining computational fluid dynamics (CFD) and MRI. Velocity patterns occurring in the ascending aorta were examined with geometrical data obtained by MRI measurements. Ramuzat et al. [25] performed PIV measurements to investigate the flow field downstream of the heart valve prostheses. The aortic valve model was manufactured from a solid acrylic block, and was polish clear for optical access. The fluid used has a matching refractive index as that of acrylic. These principles are adopted in our PIV analysis.

\section{Methodology: Fabrication of an anatomically correct, compliant model of the aorta suitable for PIV analysis}

\subsection{Requirements of the Compliant Aorta Model}

The conditions to be met by this model are threefold:

Applicable for PIV analysis: Since in PIV, light sheets generated by laser pulses are used to illuminate the particles in the fluid, an optically clear material must be used for its construction.

Anatomically correct: To accurately reconstruct the complex geometries of the aorta model, MRI data is used as a source of geometrical information. As the MRI cannot scan the aortic sinuses in detail, their dimensions were obtained from literature sources. The parts of the aorta to be recreated are shown below in Fig. 1.3 


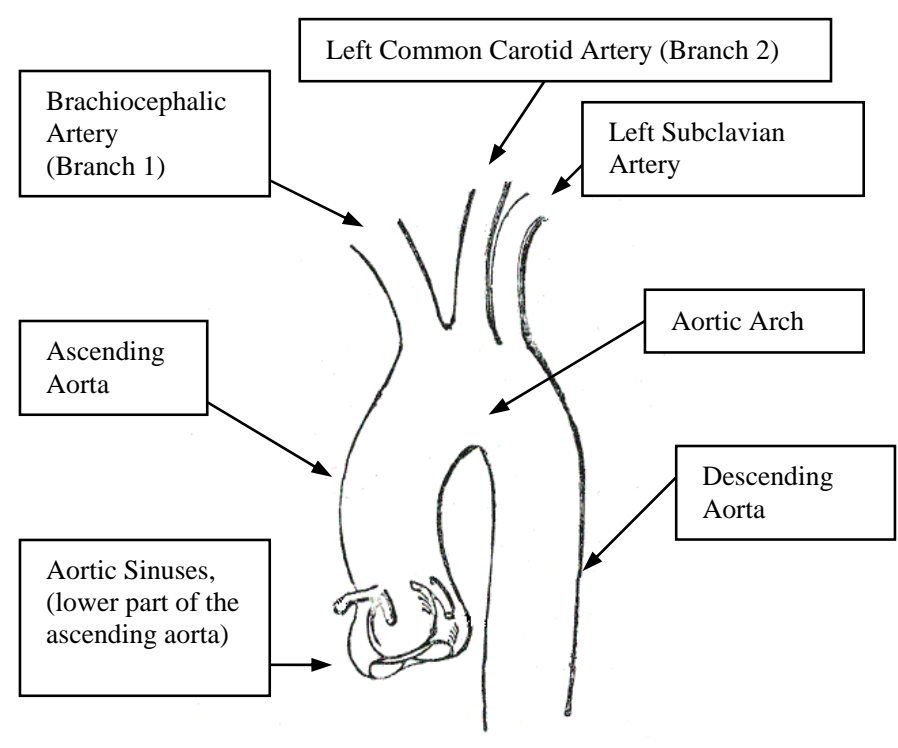

Figure 1 The anatomy of the aorta to be recreated in the model [12].

Compliant: To replicate the compliant behavior of the natural aorta, an elastic material must be used. The model is required to contract and expand passively under simulated ventricular action. The material has to be strong enough to withstand the continuous rhythmic deformations.

\subsection{Principles of Fabricating the Compliant Model}

MRI data of the aorta is obtained as a series of images. Each image shows a cross section or a "slice" of the aorta. The combination of images stacked together in sequence with the correct spacing provides a three dimensional structure of the aorta. The reconstruction of a three dimensional model from the series of two dimensional images is accomplished by the use of a software called 3D Doctor. This program is capable of importing files from the MRI and exporting files in a variety of formats recognizable by most common CAD packages. Because the MRI cannot scan the aortic sinuses in detail, their 3D modelling are accomplished with the aid of Pro Engineer based on a prior knowledge of the structures. This program is also used to correct the global structures imported from 3D Doctor. The completed CAD model is then converted to the stereolithographic (STL) file format so that a physical reconstruction can be created using a rapid prototyper.

A water soluble thermoplastic material is used as the building block for the physical reconstruction. Because the surface of the model has irregularities (the roughness is depended on the nozzle size of the rapid prototyper), a method to smooth the surface is applied. Once the model is sufficiently smooth, it is coated with an optically clear silicone one layer at a time until a desired wall thickness has been attained. After the silicone has cured, the thermoplastic core is dissolved with a basic solution. The result is an elastic, optically clear structure of the aorta that is anatomically faithful, satisfying the requirements defined in the previous section. In the following sections, detailed discussions of the each procedure of the fabrication process are provided.

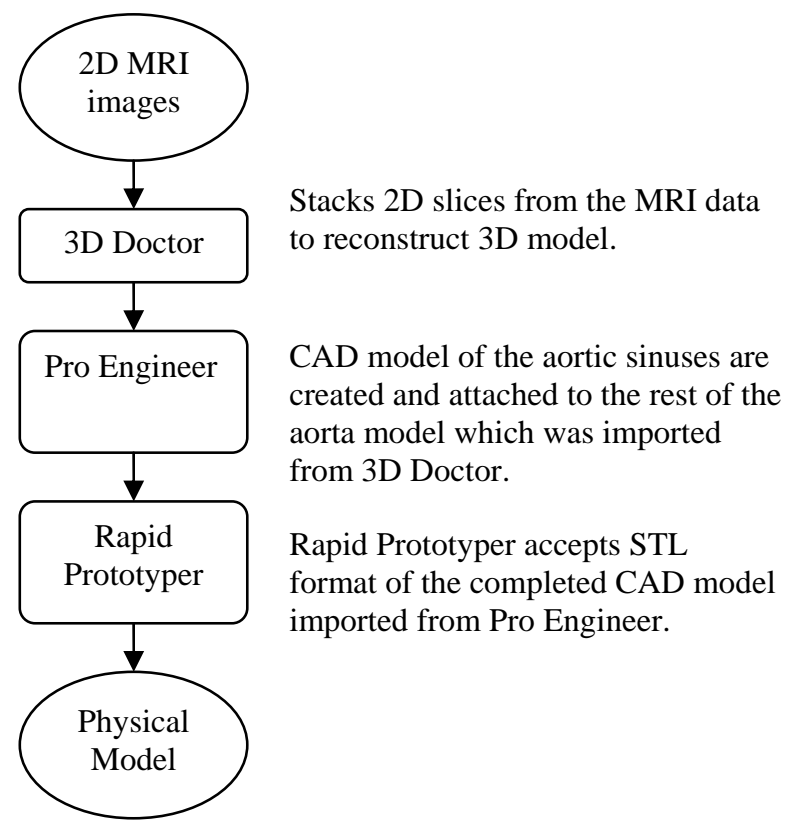

Fig. 2. Sequence in developing a 3D physical model based on 2D MRI data

\subsection{Generating the Thermoplastic Aorta Model}

Several steps are required to transform 2D MRI images into a 3D physical model.

Magnetic Resonance Imaging: For this study, cross sectional MRI images of the aorta are obtained from routine clinical acquisition. The series of 2D images in sequence with the correct spacing allows the recreation of its 3D structure. Two different sequences of images are captured: one along the vertical planes, and one along the horizontal planes. These files are stored in DICOM (DCM) format, a standard for medical images.

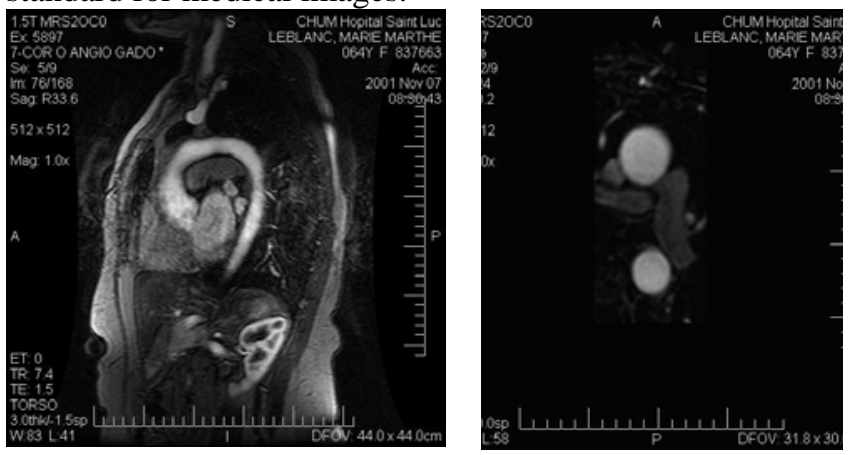

Figure 3 MRI images of the aorta along different vertical and horizontal planes 
Image Segmentation: The software 3D Doctor form Able Software Corp., is used to segment the 3D MRI dataset. It is also used to translate the medical Dicom format into CAD stardard formats (IGES, VRML). After segmentation and update (based on the clinician input), the following 3D rendering is obtained from the MRI raw data.

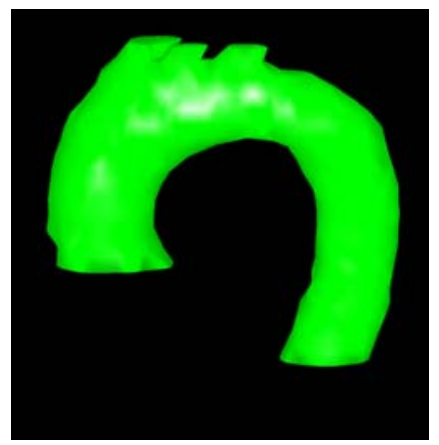

Fig. 4 Model of the aorta processed from 3D Doctor

The 3D surface is exported as a VRML file. This file format is recognizable by Pro Engineer, which is utilized for further model manipulations.

CAD File Generation: Pro Engineer is required because the aorta model constructed from 3D Doctor is incomplete and needs further correction to be anatomically correct. For example, we see in Figure 4 that the aortic sinuses (bulging) are not present below the ascending aorta of the 3D model. This is due to the inability of the MRI to differentiate overlaid anatomical structures with the same properties. The basic shapes of the aortic sinuses and their relations to the aortic valve are shown in Figure 5. The aortic valve consists of 3 leaflets, each of which is attached to the aortic root from one commissure point along the aortic ring towards another commissure point [8]. The three sinus cavities are located directly behind each of their leaflets. The geometries of sinuses for the aorta model are reconstructed with the aid of Pro Engineer.
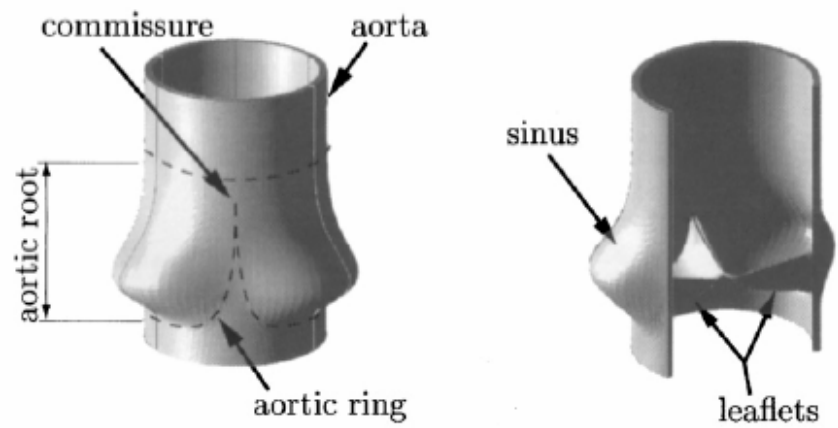

Figure 5 The aortic sinuses (left); dissection of the sinus wall with its corresponding leaflet (center); top view of the sinuses with their leaflets (right) [8].

The modelling of the aortic sinuses has previously been documented by De Hart et al. [8] in their computational analysis of the fluid-structure interaction in the aortic valve system. Several dimensions were used in their study to describe the aortic valve and sinus:
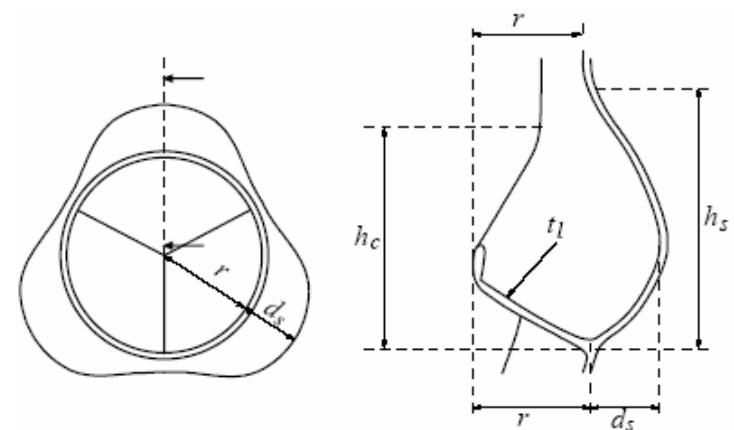

Figure 6 Relevant dimensions of the aortic valve and sinus model: $r$ denotes valve radius; $d_{s}$ the sinus depth; $h_{s}$ the sinus height; $h_{c}$ the commissure height; and $t_{l}$ the leaflet thickness [8].

\begin{tabular}{|l|l|l|l|l|}
\hline$r(\mathrm{~mm})$ & $d_{s}(\mathrm{~mm})$ & $h_{s}(\mathrm{~mm})$ & $h_{c}(\mathrm{~mm})$ & $t_{l}(\mu \mathrm{m})$ \\
\hline 12.0 & 5.75 & 21.0 & 10.5 & 200 \\
\hline
\end{tabular}

Table 1 Geometric properties of the aortic valve and sinus model [8].

Based on these dimensions, the aortic sinuses are carefully reconstructed as solid objects, i.e. with their cavities filled in (Fig.ure 7). The internal aortic valve is not considered at this moment. The modelling of the aortic valve will be implemented after the final silicone aorta model is obtained. The completed sinus reconstructions are to be connected with the aorta model which had previously been generated in 3D Doctor.

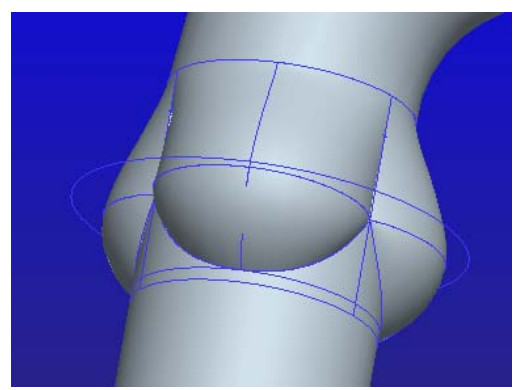

Figure $7 \mathrm{CAD}$ modelling of the aortic sinuses as solid objects in Pro Engineer

The entire model is then rescaled so that the ascending aorta has a diameter of 1 ". This is necessary to ensure a smooth connection between the ascending aorta and the aortic sinuses (with standard couplings). The following additional steps were performed:

1. The three branches above the aortic arch are extended. At the end of each branch extension, a cylinder with a slightly larger diameter is added, 2. A circular cylindrical extension is added from the base of the aortic sinuses and $\mathbf{3}$. The descending aorta was made to converge to a circular cylinder near its end (Figure 7). 


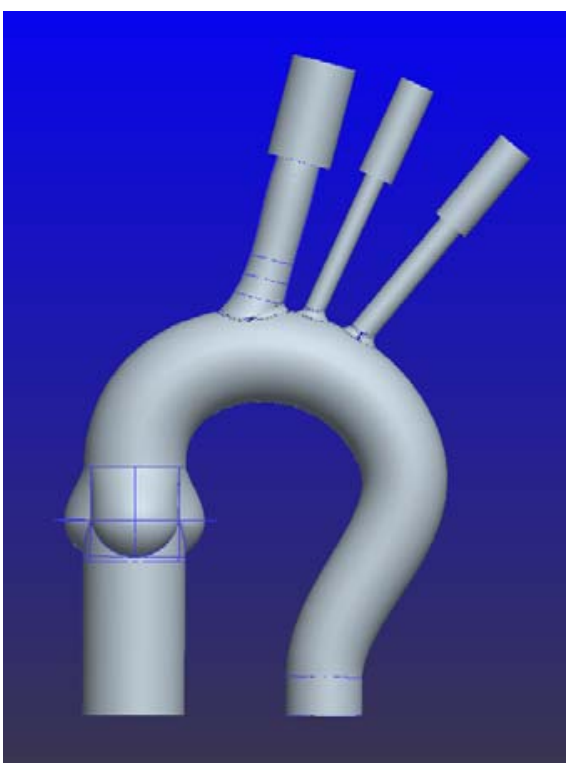

Figure 7 3D CAD model of the aorta in Pro Engineer

These modifications were applied to the CAD model so that the eventual silicone aorta model can be connected to external tubes. As well, the aortic sinuses extension was implemented to allow for the future insertion of an artificial aortic valve.

After the 3D CAD modelling is completed in Pro Engineer, the aorta is saved as a stereolithographic (STL) file. This is the required format for rapid prototyping purposes.

Rapid Prototyper: In this study, the method of RP used is called fusion deposition modelling (FDM). The Stratasys FDM 3000 Rapid Prototyper is used for the fabrication of the aorta model. The maximum part volume that can be created is 9.5 " by 9.5 " by 16 " and it has an achievable accuracy of +/- 0.005" [18]. The system uses AcrylonitrileButadiene-Styrene (ABS) or ABSi (for high impact) thermoplastic as the build material. The support structure is created from a water soluble thermoplastic called WaterWorks. This material has the interesting ability to dissolve in a special water based solution. Therefore, even if the support material is located in far hidden regions of the part, it can simply be washed away by immersing the part in the solution. The process of dissolving the WaterWorks support can be accelerated by placing the part in an ultrasonic tank filled with the required solution. (Figure 8).

The roles of the ABS and WaterWorks thermoplastics are reversed in the fabrication of the aorta model. WaterWorks is used as the build material. ABS will act as its scaffolding. This modification is done easily by the FDM software. Recall, silicone will be coated over the thermoplastic aorta model. Once the silicone has cured, the thermoplastic must be removed in a fashion that leaves the silicone unharmed. Using WaterWorks as the build material allows for its eventual removal without damaging the silicone.

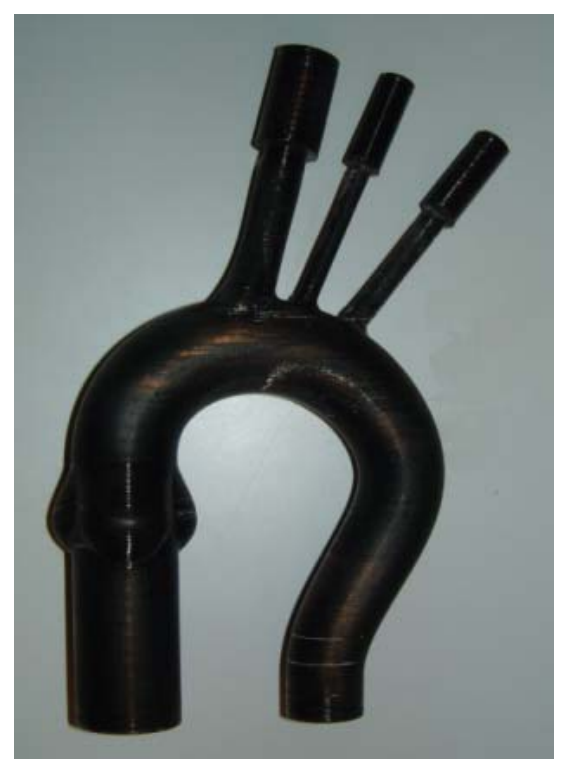

Figure 8 Rapid prototyping results from the FDM process

\subsection{Fabricating the Compliant Aorta Model}

Silicone from Dow Corning (SYLGARD 184) was used to manufacture the compliant aorta model. This product is often used to protect parts against mechanical and thermal shock [9].

The silicone kit consists of a base and a curing agent. To apply this product, the two liquid components are mixed thoroughly using a weight or volume ratio of 10:1and subsequently thermally cured. A hybridization oven (Fisher Scientific Isotemp P120) was modified to hold and cure the aorta model. For this model, a curing temperature of 45C during 10 to 12 hours was used. Several earlier tests showed that any higher temperature create swelling in the silicone.

The liquid form of SYLGARD 184 silicone was applied uniformly over these pieces until a layer thickness of 1/4" has been developed. Once the silicone has cured, the WaterWorks core is then dissolved leaving the silicone shell. Once the WaterWorks is dissolved we get the compliant anatomically correct model presented in the following figure.

The resulting aorta model satisfies all the requirements. The optically clear property of the silicone makes this model compatible for PIV analysis. 2) The model (based on MRI data and aortic sinus dimensions from De Hart et al. [8]) is anatomically correct. 3) The elastic nature of silicone enables the model to expand and contract passively under simulated ventricular action, i.e. the model is compliant. 


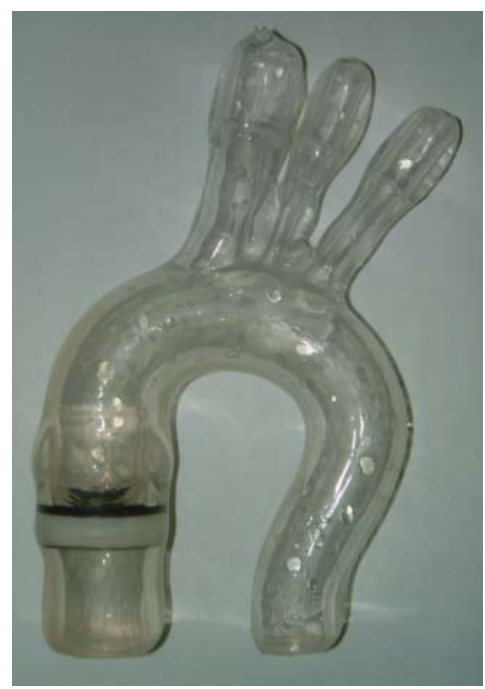

Figure 9 Anatomically correct transparent model of the aorta made from Silicone

\section{Hydraulic model with the Compliant Aorta Model for PIV Analysis}

\subsection{Artificial Aortic Valve}

Found between the aorta and the left ventricle, the aortic valve contains three leaflets that function as a one-way valve. As the left ventricle contracts, this valve allows blood to flow into the aorta. The aortic valve then closes as the heart relaxes between contractions, preventing blood in the aorta from returning to the left ventricle. In order to accurately recreate this flow behavior for the silicone model during simulated ventricular action, an artificial valve has to be installed prior to PIV analysis. Mechanical valves can be made of metal, plastic or other synthetic materials. One commonly used mechanical valve is the St. Jude's valve. This bileaflet design consists of two semi-circular discs that pivot on hinges. The discs open with each contraction of the left ventricle and close when it relaxes. In the following figure, we show a close-up of the aortic root with the valve inserted.

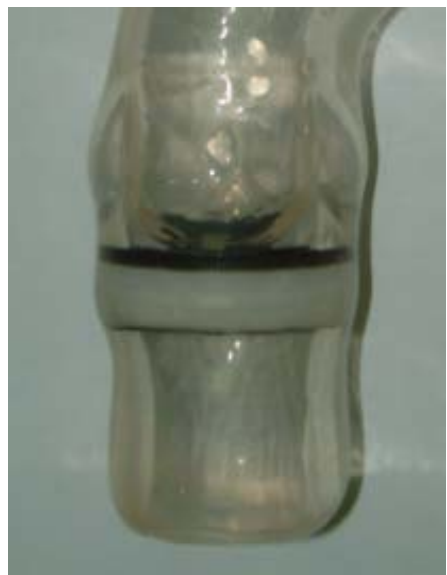

Figure 10 (a) Mechanical bileaflet (St. Jude's) valve inserted just below aortic sinuses

\subsection{PIV Viewing Box}

A chamber is required for the PIV acquisition in order to avoid refractive index matching problems (reflections, refractions). A simple box was generated around the model. Holes on this box were required so that support tubes for the silicone model can be installed into the chamber. The locations of these holes were designed in a way that any support tubes connected to the model would not apply unnecessary stress to the silicone branches, aortic sinuses extension, and descending aorta.

The top was left open to allow for quick access to the silicone model. A lid was needed that would keep the chamber liquid tight. The final design is shown in Figure 11. Because the model was to be used for PIV analysis, the entire chamber was built with transparent acrylic.

To install the silicone aorta model into the chamber, its aortic sinuses extension and descending aorta were fitted over the 2 inner protruding acrylic tubes of the lower wall panel. Three flexible tubes of dimensions (5/8" OD, 1/2" ID), (5/16” OD, 3/16” ID), (3/8” OD, 1/4” ID) were inserted through the 3 outer protruding acrylic tubes of the upper wall panel (from left to right). These tubes were then drawn and fitted into the branch extensions of the aorta model. Finally, 2 flexible tubes of dimensions ( 1 1/4" OD, 1 " ID) and (1" OD, 3/4" ID) were connected to the outer protruding acrylic tubes of the lower wall panel. Note that all connections were tightened with metal ring clamps to prevent leakage.

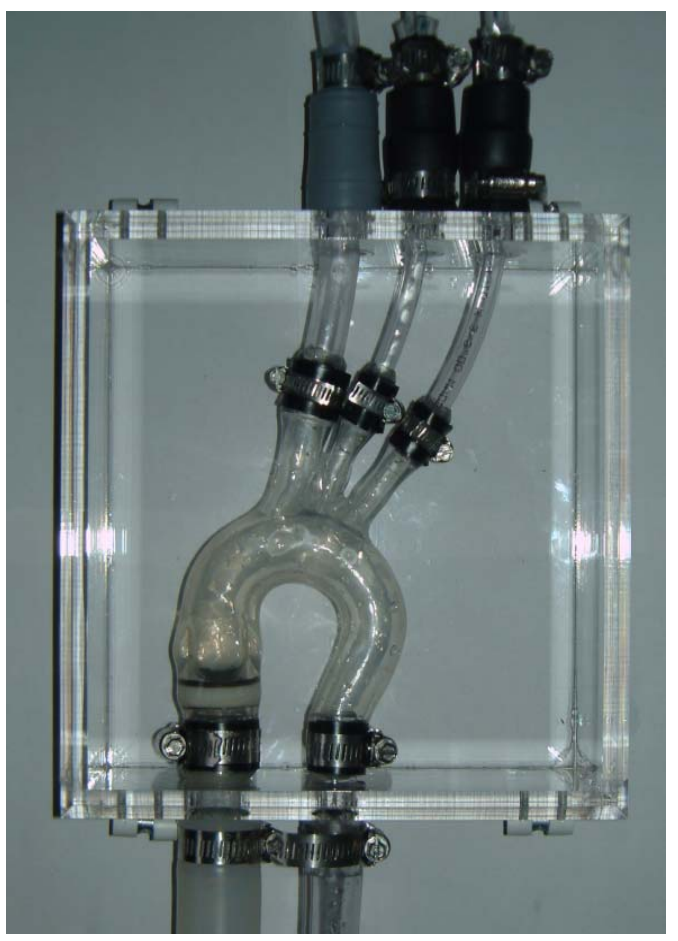

Figure 11 PIV acrylic viewing box 


\subsection{Pulsatile Pump Implementation}

The cardiac cycle is the sequence of events that occurs during each heart beat. It consists of 2 phases: diastole and systole. To simulate the contraction and relaxation of the left ventricle, a pulsatile pump (Harvard Apparatus; model 1423) was used to circulate fluid through the aorta model. This pump mimics the cardiac cycle by the relying on a piston actuator and ball check valves to provide the proportioning action [14]. Diastole is achieved by the backward movement of the piston, which fills the piston cylinder (left ventricle) with fluid. Systole occurs as the piston is moved forward. The amount of fluid displaced during each stroke motion represents the stroke volume. Back flow during either phase is prevented by the ball check valves.

\subsection{Working fluid}

Once the aorta model was connected to the pulsatile pump and the reservoir with the appropriate tubes, the system needed to be primed with a working fluid. Because this aorta model was to be used for PIV analysis, the silicone must become almost "invisible" when in operating conditions [5].

When submerged in a liquid, the silicone model, even though it is optically clear, does not normally become invisible. This is due to the fact that the silicone and the liquid do not, in general, have matching refractive indexes. According to the Law of Refraction, the image (light) of the object gets refracted as it travels through the different media (silicone and liquid) [13]. It is this distortion that allows the viewer to perceive the silicone model; rendering it visible, even though it is optically clear. These distortions are unacceptable in PIV experiments because the image of the particles within the model must remain undistorted to produce accurate velocity measurements. Also, if the liquid and the model do not have matching refractive indexes, the direction of the illuminating laser sheet can potentially be deflected as it travels through the silicone [5]. It is for these reasons that the model must be circulated with a fluid having a matching index of refraction as that of the silicone used. The quiescent fluid in which the model is submerged must also have that same property. (Note that the refractive index of the acrylic chamber is irrelevant because its flat panels remain perpendicular to the laser light sheet and camera direction at all times [23].)

Mixtures of glycerin and water at different proportions produce clear and colorless fluids of varying refractive indexes. These indexes are also temperature dependent. Their relationship was described by Nguyen et al. [23] as follows:

$$
n_{\text {target }}=p\left(a_{1}+b_{1} T\right)+(1-p)\left(a_{2}+b_{2} T\right)
$$

where $n_{\text {target }}$ is the refractive index of the mixture, $T$ is the temperature in Kelvin, $p$ and (1-p) are the proportions of glycerin and water, $\left(a_{1}=1.3774, b_{1}=-0.0002\right)$ and $\left(a_{2}=\right.$ 1.5463, $\left.b_{2}=-0.0002\right)$ are empirical constants specific to glycerin and water.

Silicone used for the aorta model (SYLGARD 184) has a refractive index of 1.414 [6]. By setting $n_{\text {target }}=1.414$ and $T$ $=298 \mathrm{~K}, p$ was evaluated as 0.43 . Therefore, to match the refractive index of silicone at room temperature, a mixture of $43 \%$ glycerin to $57 \%$ water was used as the working fluid in this study.

\section{PIV Analysis of Compliant Aorta Model}

\subsection{PIV System Components}

Flow visualizations of the aorta model were acquired by utilizing a PIV system from TSI. It consists of the following components [35]:

- New Wave Research Solo Nd:YAG laser provides the illumination of the flow field.

- $\quad$ TSI Model 630047 PIVCAM 13-8 Image Capture System (CCD Camera)

- TSI Model 610034 LaserPulse Synchronizer controls the timing of the laser and the camera, ensuring each component operates in the correct sequence.

- Windows NT based Pentium III computer contains Insight Software which analyzes PIV images and computes flow field parameters.

\subsection{Aorta Model Setup}

The chamber of the aorta model was oriented in such a way that the laser light sheet would first penetrate the aortic sinuses and the ascending aorta. To ensure that the compliant aorta does not collapse during the diastolic phase of the cardiac cycle (when fluid pressure is at its lowest), the reservoir was raised and put on a platform to increase the hydrostatic pressure within the aorta model. The glycerin/water mixture was seeded by adding titanium oxide $\left(\mathrm{TiO}_{2}\right)$ particles into the reservoir. The fluid was stirred from the reservoir for several minutes while the pump was running to adequately mix the particles. Finally, all lustrous metallic parts of the setup (metal screws, ring clamps, etc.) were covered with flat black tape. This made sure that the intense laser does not damage the camera by reflecting light off of these large metallic surfaces.

\subsection{Data Acquisition Methods}

In the first flow imaging experiment, the aortic sinuses were observed. Having properly adjusted the camera and laser positions, and all of the necessary settings in the Insight Software, 30 pairs of image captures were made at a pulse repetition rate of $5 \mathrm{~Hz}$. This rate specifies the start of one laser pulse sequence to the start of the next laser pulse 
sequence [36]. By capturing 30 sequences of images, it ensures that different periods of the diastolic and systolic phases can be visualized. The pulsatile pump was fixed to the following settings: $40 \%$ phase ratio (percentage of systole in the cardiac cycle), 50 beats/min, $80 \mathrm{~mL} /$ stroke. During this simulated ventricular action, it was noted that the volume change in the aorta model was approximately $0.6 \mathrm{in}^{3}$, deduced from the fluid height change in the vertical acrylic tube. This value can be adjusted by changing either the phase ratio or stroke rate. The following figure shows a pair of images captured during one of the laser pulse sequences. Pulse separation value was set to 200 microseconds. This is the time between the two laser pulses (or image captures) of each sequence.
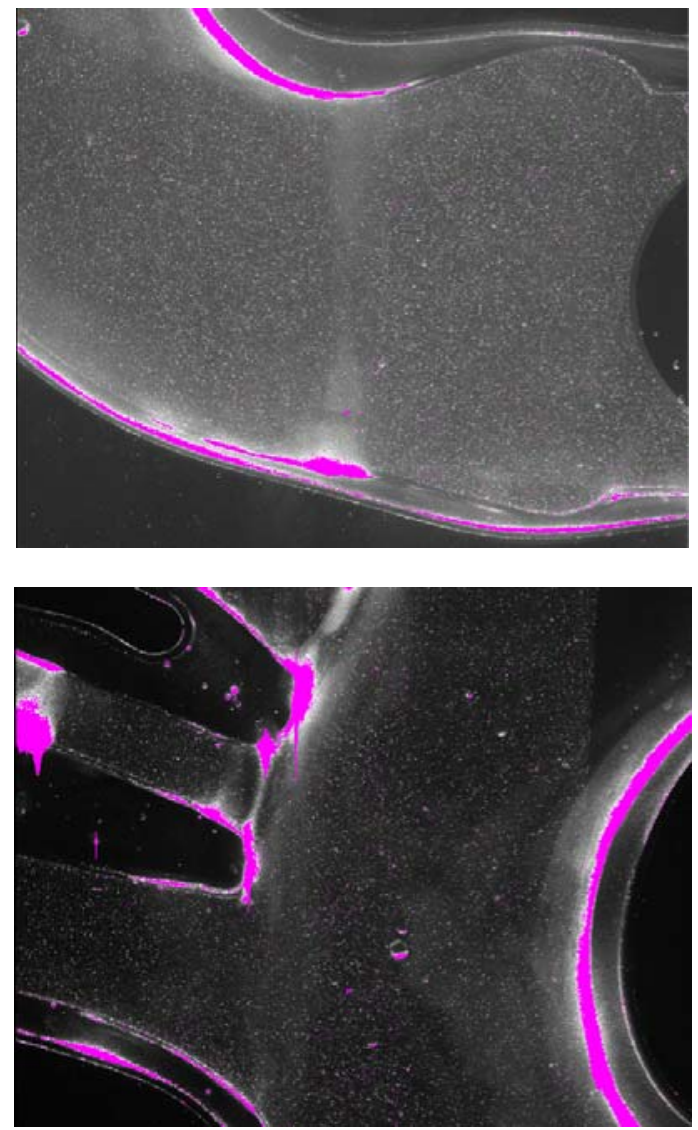

Figure 12 Raw images of the aortic root (top) and side branches (bottom) captured by the PIV camera in one laser pulse sequence (The round shaped object to the right is one of the leaflets of the mechanic aortic valve).

Velocity calibrations were previously made by defining a known distance (the sinus diameter) in a selected image. The raw velocity vectors (Figure 13a) were smoothed, filtered and validated to reduce spurious velocities (Figure 13b).
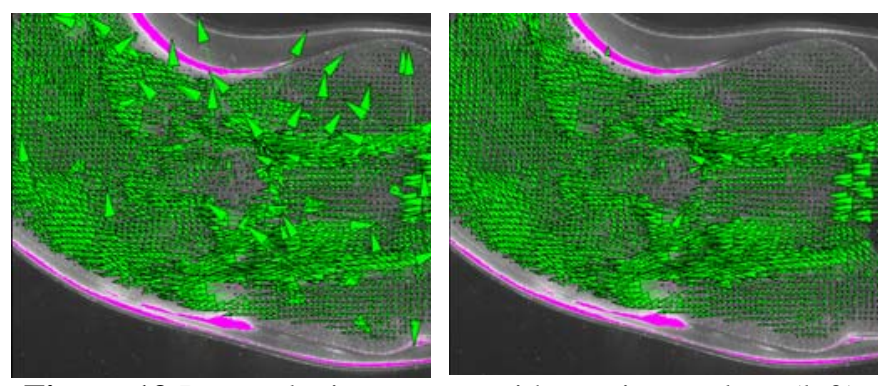

Figure 13 Raw velocity vectors with spurious values (left) and processed validated velocity vectors (right)

\subsection{PIV data post-processing}

The two areas of Figure 12 of the compliant model were imaged: the aortic sinuses and the aortic arch (side branch). For each region, 2 imaging runs were conducted under different pump parameters. Thirty image sequences were captured at a pulse repetition rate of $5 \mathrm{~Hz}$ for each of these runs to ensure different phases of the cardiac cycle could be observed. Selected results are presented below with the aid of Techplot.
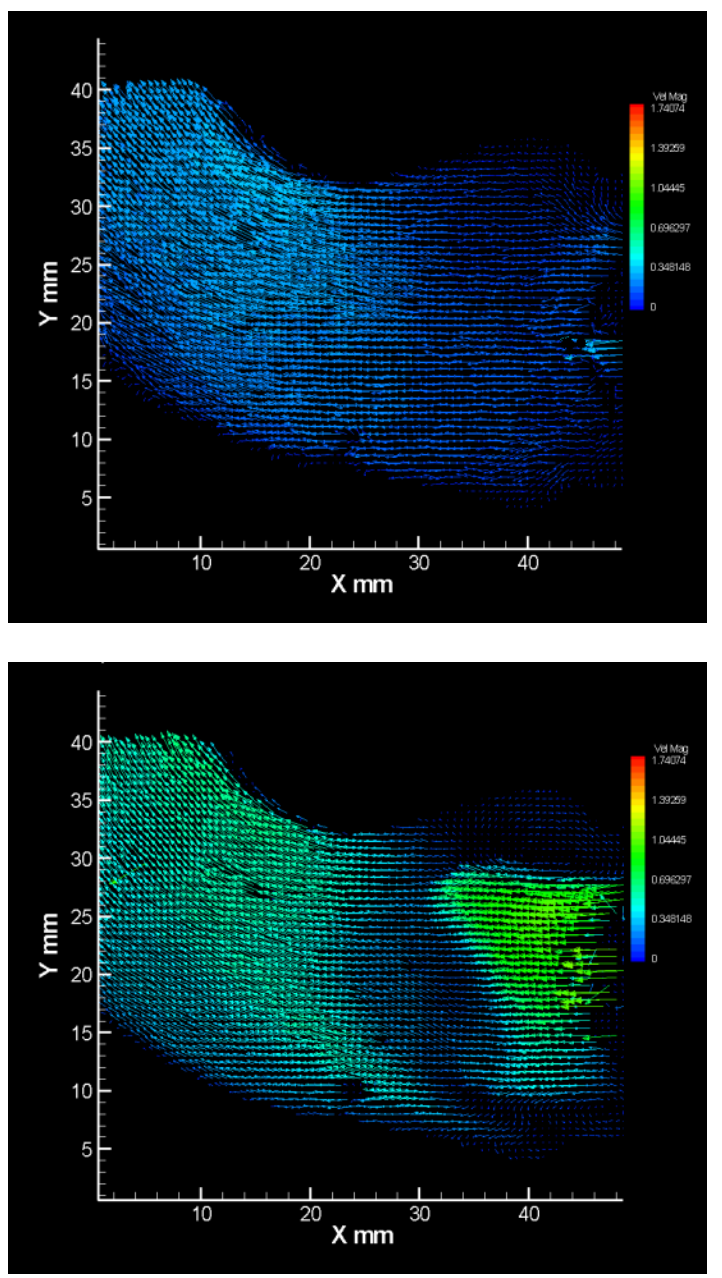

Figure 14 Velocity distribution in the aortic root at diastole (top) and systole (bottom). 
The velocity scale for the reported PIV acquisitions is given in the following figure in order to simplify the subsequent figures

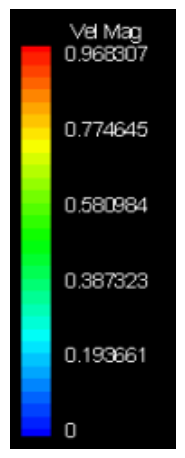

Figure 15 Velocity scale of the PIV acquisitions
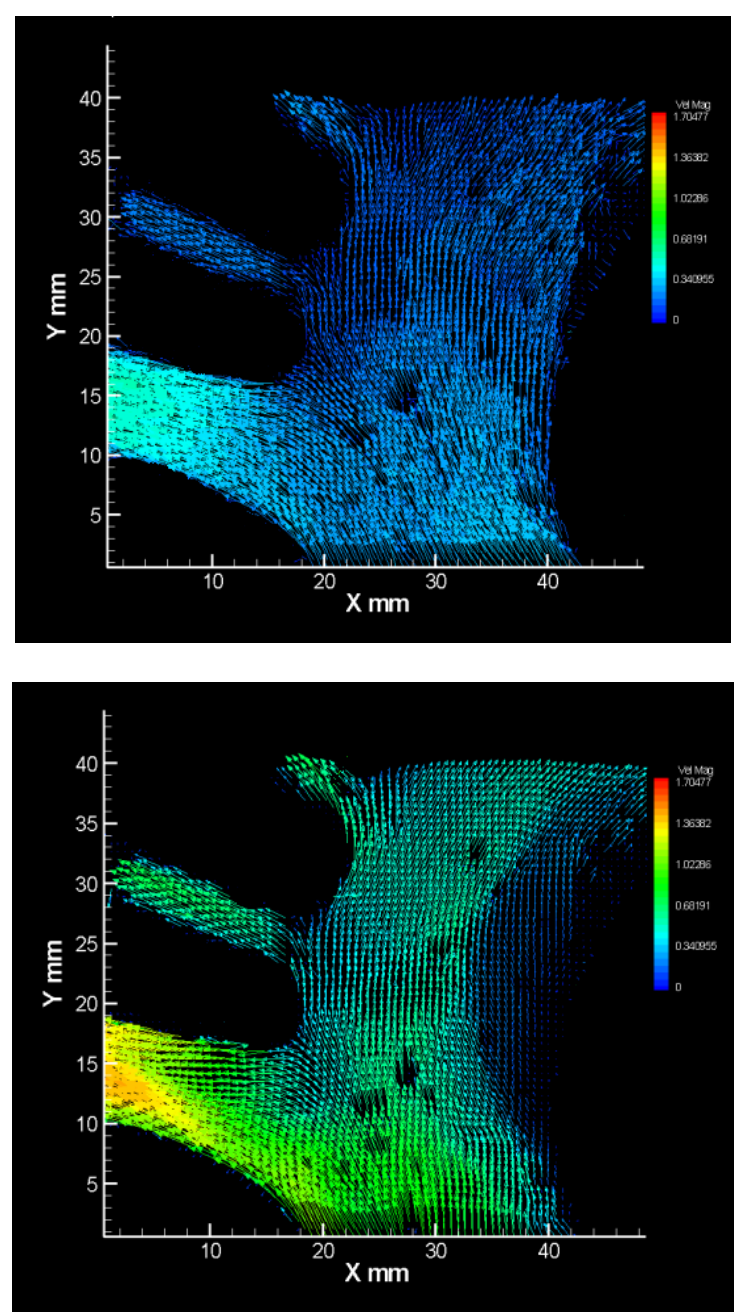

Figure 16 Velocity distribution in the side branches at diastole (top) and systole (bottom).

Vortex shedding can be observed during diastole in all 3 regions of interest. This is especially noticeable in the aortic sinuses flow visualizations. On the other hand, flows were much more uniform at the systolic phase. In general, the velocities were greater in the aorta during systole because the phase ratios were set to less than $50 \%$ (forward stroke of the pump is faster); also, the aortic valve prevents flows in diastole to speed up.

Because this study was in part a feasibility test, a number of parameters had yet been optimized. For the fluid used in the flow visualization experiment, no attempt was made to match the kinematic viscosity $(v)$ of blood. This could affect the Reynolds Number $(\operatorname{Re} \propto 1 / v)$, which is an important factor to consider when recreating the flow dynamics of the aorta. Blood analogue for optical flow experiments is typically composed of $36.7 \%$ glycerin in water to match the kinematic viscosity [23]. Afterwards, the refractive index is adjusted by adding components such as sodium thiocyanate (NaSCN), potassium thiocyanate (KSCN), or zinc iodide (ZI) [23]. Because these additional components are potentially toxic or flammable, none of them were used for this initial study. Instead, only the refractive index of silicone was taken into consideration by increasing the proportions of glycerin to $43 \%$ in the fluid.

It should be noted from the flow visualizations of the aortic arch that a number of holes existed where no vectors are present. This is due to small air pockets that were not removed or degassed during the silicone curing process in the fabrication stage of the aorta model. Because a clear water based acrylic paint was used for the smoothing process, it was at times difficult to perceive the tiny trapped air bubbles.

\section{Conclusion}

By developing an innovative approach of manufacturing an anatomically correct, transparent and compliant aorta model, the initial goals were achieved. Indeed an anatomically realistic model, which included the aortic sinuses, ascending aorta, aortic arch, and descending aorta, was developed in a one-to-one scale. Local variation in compliance was taken into account. Furthermore, its transparent nature made it suitable for PIV imaging techniques. In previous studies of aorta mechanics, models of the ascending aorta fluid flow have been idealized as rigid curved vessels; very few compliant models have been investigated. In fact, no models including the aortic sinuses, ascending aorta, and aortic arch have been examined. To the best of our knowledge, the model fabricated in this study is the first of its kind to include all of these structures. The capability of the aorta model for PIV analysis was verified allowing to observe complex flow structures in the aorta.

Some parameters have yet to been optimized. As a result, several improvements to the flow visualization experiment can be made. For the working fluid, no attempts were made to match the blood kinematic viscosity. The correct viscosity can be attained by adding components such as sodium thiocyanate (NaSCN), potassium thiocyanate, or zinc iodide (ZI) to the glycerin/water mixture in proper proportions. However, the potentially hazardous nature of 
the resulting fluid requires the user take caution. The internal fluid pressure of the aorta also needs to be adjusted. By appropriately raising or pressurizing the connected fluid reservoir, the pressure within the model can be set to the desire value. Finally, to achieve complete transparency of the model, the silicone should be rid of all trapped air pockets. Any remaining pockets of air can cause flow particles to be obscured. Although most of the bubbles were removed during the fabrication process, some smaller ones were remained undetected.

The model has the potential to be used to validate numerical simulations with complex fluid structure interaction flow physics. The model also has the potential to be used to assess the hydrodynamic performance of cardiovascular devices (shear stress levels, pressure gradients). This possibility is also significant in view of reducing the number of animals used in in-vivo experiments.

\section{Refrences}

1. Able Software. 3D Doctor Tutorial http://www.ablesw.com/3d-doctor/tutor.html

2. Advanced Ceramics Research. Aquacore Specification Sheet [online]. Available: http://www.acrtucson.com/products/Aquacore/spe csheet.htm [Accessed Feb. 2004]

3. Babl FE. Household bleach. Clinical Toxicology Review 1997; 19, No. 4.

4. Cardiovascular Consultants of Maine. Aortic Stenosis, Treatment, .http://www.heartmaine.com

5. Chow YC, Uzol O, Katz J, Meneveau C. An investigation of axial turbomachinery flows using PIV in an optically-unobstructed facility. The 9th International Symposium on Transport Phenomena and Dynamics of Rotating Machinery. Honolulu, Hawaii, Feb. 10-14, 2002.

6. Clingan PA, Friedman MH. The effect of celiac and renal artery outflows on near-wall velocities in the porcine iliac arteries. Annals of Biomedical Engineering 2000; 28: 302-308.

7. Dantec Dynamics. Measurement Principles of PIV http://www.dantecmt.com/PIV/Princip/Index.html

8. De Hart J, Peters GWM, Schreurs PJG, Baaijens FPT. A three-dimensional computational analysis of fluid-structure interaction in the aortic valve. Journal of Biomechanics 2003; 36: 103-112.

9. Dow Corning. SYLGARD ${ }^{\circledR} 184$ Silicone Elastomer Production Information.

10. Fisher Scientific. Isotemp ${ }^{\circledR}$ Hybridization Incubators Operation Manual.

11. Fox RW, McDonald AT, editors. Fluid Statics. In: Introduction to Fluid Mechanics. 4th ed. New York: John Wiley \& Sons, 1992: 50-95.

12. Gray’s Anatomy. The Aorta [online]. Available: http://www.bartleby.com/107/142.html

13. Halliday D, Resnick R, Walker J, editors. Geometrical Optics. In: Fundamentals of Physics,
Extended. 4th ed. New York: John Wiley \& Sons, 1993: 1011-1048.

14. Harvard Apparatus, Inc. Series 1400 Pulsatile Blood Pumps User's Manual.

15. Holman JP, editor. The Design of Screws, Fasteners, and Connections. In: Mechanical Engineering Design. 5th ed. New York: McGrawHill, 1989: 325-381.

16. Klabunde RE. Vascular Compliance, Cardiovascular Physiology Concepts: http://www.cvphysiology.com/Blood\%20Pressure/ BP004.htm

17. Leuprecht A, Perktold K, Kozerke S, Boesiger P. Combined CFD and MRI study of blood flow in a human ascending aorta model. Biorheology 2002; 39: 425-429.

18. Materialise. Fused Deposition Modelling: http://www.materialise.be/prototypingsolutions/fus ed_ENG.html

19. Moore JA, Steinman DA, Ethier CR. Computational blood flow modelling: Errors associated with reconstructing finite element models from magnetic resonance images. Journal of Biomechanics 1998; 31:179-184.

20. Moore JA, Steinman DA, Holdsworth DW, Ethier CR. Accuracy of computational hemodynamics in complex arterial geometries reconstructed from magnetic resonance imaging. Annals of Biomedical Engineering 1999; 27: 32-41.

21. Morris D. Removing Paint: http://www.gremlins.com/david_morris/paint_rem oval.html

22. National Heart, Lung, and Blood Institute (NHLBI). Morbidity \& Mortality: 2002 Chart Book on Cardiovascular, Lung, and Blood Dieases http://www.nhlbi.nih.gov/resources/docs/02_chtbk .pdf

23. Nguyen TT, Mongrain R, Brunette J, Biadillah Y, Tardiff JC. A method for matching the refractive index and kinematic viscosity of a blood analogue for PIV investigations in hydraulic cardiovascular models. Journal of Biomechanics 2004 [in print].

24. Partain CL. Magnetic Resonance Imaging. Microsoft Encarta Encyclopedia 2004.

25. Ramuzat A, Browne P, Yoganathan AP. Flow field measurements downstream of a heart valve by means of particle image velocimetry technique. 1999 Bioengineering Conference. Big Sky, Montana, June 16-20, 1999.

26. Reul H. Hydraulic analog model of the systemic circulation - designed for fluid mechanical studies in the left heart and systemic arteries. Advances in Cardiovascular Physics 1983; 5: 43-54.

27. Rikert L. Stripping paint from styrene and resin plastic models: http://pw1.netcom.com/ lrickert/On30/FAQ/Other/FAQstrip.html

28. Shahcheraghi N, Dwyer HA, Cheer AY, Barakat AI, Rutaganira T. Unsteady and three-dimensional simulation of blood flow in the human aortic arch. 
Journal of Biomechanical Engineering 2002; 124: 378-387.

29. St. Jude Medical. Heart Valve Replacement: http://www.sjm.com

30. Stanford University Medical Center. Cardiovascular Diseases: Aortic Stenosis: http://www.lpch.org/DiseaseHealthInfo/HealthLib rary/cardiac/as.html

31. Stratasys, Inc. The FDM Process: http://www.stratasys.com/NA/index.html

32. Sundt TM. Aortic Valve Replacement: http://www.sts.org/doc/3620

33. Swanson MB, Davis GA, Perhac DG. (1995) Environmentally Preferable Cleaners,: http://eerc.ra.utk.edu/ccpct/pdfs/EnvPrefCleanerswholedoc.pdf

34. The Painting Clinic. Paint Stripping Page: http://www.paintingclinic.com/clinic/guestarticles/ removingpaint3.htm

35. TSI, Inc. PIV System Installation Manual.

36. TSI, Inc. INSIGHT ${ }^{\mathrm{TM}}$ Particle Image Velocimetry Software (Version 3.3) Instruction Manual. 